\title{
LA MOTIVACIÓN EN LOS DIFERENTES CONTEXTOS EDUCATIVOS EN ALUMNADO DE EDUCACIÓN SECUNDARIA OBLIGATORIA
}

Ceciliano Gil, Mirian; Feria Acosta, Laura; González Caracuel, Jenny y Orta Torilo, Soraya. Universidad de Huelva

\section{RESUMEN}

En el presente artículo realiza una revisión teórica sobre la importancia que ejerce la motivación en el aprendizaje de los alumnos de Educación Secundaria Obligatoria. En primer lugar, se realizará un breve análisis sobre los cambios, tanto físicos como psicológicos, que influyen en los adolescentes y en su motivación para aprender los contenidos teóricos y prácticos durante su estancia en el instituto. Posteriormente, se realizará un análisis sobre los factores que más influyen en esta motivación, tales como el profesor, los iguales y el contenido que se pretende enseñarles. Las conclusiones extraídas indican que las últimas reformas educativas se han centrado en potenciar la motivación académica y las calificaciones escolares de los estudiantes, sin profundizar en el conocimiento de la influencia que pueden tener los factores sociales o contextuales sobre la motivación en el ámbito escolar.

PALABRAS CLAVE: motivación, adolescentes, profesorado de secundaria, grupo de iguales, contenido curricular.

\section{INTRODUCCIÓN}

En el presente artículo se pretende realizar una revisión teórica de las influencias del profesorado, de los iguales y del contenido/tarea respecto a la motivación del alumnado de Educación Secundaria Obligatoria (ESO).

Dichas influencias, unidas a los cambios que se producen en la adolescencia, merecen mención; por ejemplo, se destacan los que se dan a nivel físico (comienza a salir el vello púbico, crecen las mamas de las chicas, se ensanchan las caderas, crece vello en la cara de los varones...); en definitiva, se puede decir que sus cuerpos van madurando. Además, se deben destacar los cambios psicológicos que se dan en estos jóvenes (el sentido de la vergüenza se agudiza en la adolescencia, dan más importancia lo que otras personas piensen de ellos, etc.) ya que, debido a los requerimientos académicos, tienen que madurar cognitivamente, además de los cambios físicos mencionados anteriormente.

Así, se destaca a estas edades, sobre todo al inicio de la adolescencia, los cambios que se dan en las relaciones que mantienen con otras personas, puesto que antes eran más intensas con los familiares, y ahora comienzan a ser los amigos las personas más importantes para ellos y, por tanto, también son vitales las influencias que ejercen estos en el plano académico. Además, con el paso del colegio al instituto también se producen cambios en relación a los contenidos, puesto que éstos van siendo cada vez más y a la vez más complejos. Por otro lado, los profesores son diferentes y cada docente le da por lo general una asignatura, por lo que se deben adaptar a las exigencias y requerimientos de cada docente lo cual exige, por parte de los alumnos, una capacidad de adaptación considerable. 
Dichos profesores, puede ejercer alguna influencia en la motivación que tengan los alumnos sobre el contenido, ya que puede adaptar éste a las necesidades y preferencias de los adolescentes, en la medida de lo posible. Por ello, se parte de la idea de que dichos profesores deben tener ciertas habilidades para ejercer su práctica diaria y que ésta sea lo más efectiva posible. La mayoría de estas habilidades pueden adquirirse, a pesar de que no se debe olvidar la influencia de ciertas características de personalidad.

Los cambios que se dan en los adolescentes y que fueron nombrados anteriormente, influyen en el contenido, la relación con los iguales y la influencia del profesor puesto que, al variar el contenido y la forma de presentación del mismo, así como las habilidades que tiene el docente para hacer frente y los hábitos de sus iguales, se verá de forma diferente según si los alumnos han sufrido cambios tanto físicos como psíquicos.

\section{Influencia de profesores en la motivación de los alumnos}

Los profesores desempeñan un rol fundamental en la educación. Hoy en día, para ser un buen profesor, no basta con poseer determinados conocimientos y divulgarlos, sino que un buen profesional de la educación ha de saber aplicarlos. Además, ha de estar dotado de una serie de características personales (comunicación interpersonal, asertividad, técnicas de habilidades sociales, etc.) y de habilidades docentes (métodos o estrategias de enseñanza) que favorezcan el desarrollo de su trabajo (Román, 2008).

En definitiva, el profesor que posee cualidades humanas apropiadas para la enseñanza consigue que sus alumnos estén más motivados (Bernardo, 2004). Según Bernardo (2004), algunas de las habilidades y/o competencias del profesor que facilitan la motivación del alumno son las siguientes:

- La atmósfera interpersonal de comunicación en la que se desenvuelve la tarea ha de permitir al alumno sentirse apoyado cálida y honestamente, respetado como persona y capaz de dirigir y orientar su propia acción.

- Conviene utilizar, cuando sea necesario, la represión en privado, la conversación particular amistosa y los factores positivos que animen al alumno. Son más eficaces unas palabras de ánimo, la confianza en las posibilidades de rectificación y el reconocimiento de los aspectos positivos en la conducta y en el trabajo del alumno que los castigos.

- El profesor a de mostrar interés por cada alumno; por sus éxitos, dificultades, planes, etc., y hacer que él lo note.

- El elogio es útil si surge de un modo espontáneo y se dirige a un trabajo o esfuerzo concreto.

- Por otra parte, los alumnos declaran sentirse más motivados por el profesor que prepara cuidadosamente sus clases, sabe organizar el trabajo, exige de forma razonable un rendimiento proporcionado, revisa las tareas y corrige a tiempo los exámenes, etc..

En conclusión, es esencial la mejora y formación de las características personales y habilidades sociales del profesorado para que éstos puedan ejercer de forma adecuada su actividad como docente. 


\section{Motivación de los alumnos en función de sus iguales}

Por medio de la interacción social los niños aprenden normas de conducta, actitudes, comportamientos deseados por la sociedad y aquellos instrumentos culturales necesarios para una buena adaptación al escenario socio-cultural en el que el niño se halla inmerso (Rogoff, 2003). El desarrollo social se halla intrínsecamente relacionado con el desarrollo cognitivo a lo largo de todo el ciclo vital.

En este sentido, para los adultos la amistad no es una relación unilateral en la que una persona es atraída por otra, sino implica satisfacción psicológica mutua caracterizada por la comprensión de los sentimientos y pensamientos de ambas personas, por el cuidado físico y psíquico entre los seres humanos implicados en la relación, por una comunicación íntima y sincera, por tener unas expectativas de reciprocidad en relación a los valores fundamentales de las relaciones humanas y por una estabilidad a lo largo del tiempo que trasciende los conflictos ocasionales (Damon, 1977). Todos estos aspectos que constituyen lo que es una verdadera amistad, hacen que estas relaciones sean muy valoradas y gratificantes para los seres humanos, por lo que una ruptura podría incidir muy negativamente en el desarrollo social y personal del individuo.

Damon (1989) distingue tres tipos de aprendizaje entre iguales que se diferencian, a su vez, por el grado en el que se dan dos dimensiones de la interacción: igualdad y compromiso mutuo. La igualdad hace referencia al grado de simetría que se establece entre los participantes de una situación social. Sin embargo, el compromiso mutuo alude al grado de conexión, bidireccionalidad y profundidad de las conversaciones que se establecen en la participación. Tipos de aprendizaje según Damon (1989):

- Relación de tutoría. Lo esencial de esta relación es que un niño, que puede considerarse experto, instruye a otro que puede ser considerado un novato.

- Aprendizaje cooperativo. Este entorno se caracteriza porque el grupo es heterogéneo en habilidad y los niños pueden asumir diferentes papeles.

- Colaboración entre iguales. Existe un mayor grado de mutualidad e igualdad. Las relaciones que se establecen son, generalmente, simétricas y se caracterizan por una alta igualdad y mutualidad.

Actualmente, nadie duda que no sea posible comprender y analizar el desarrollo humano sin aludir al contexto social donde se desenvuelven los seres humanos.

\section{Influencia del contenido curricular sobre la motivación.}

El contenido curricular y su estudio son de vital importancia, puesto que se sabe que influye directamente en el rendimiento y resultados en los alumnos, al igual que en los resultados como educador del profesor. Por ello, se debe conocer cómo se da este proceso en la enseñanza-aprendizaje, en la relación de feedback entre profesor y alumno.

Así, Junco, (2010) se centra en las cualidades del contenido como modelador motivacional que dependerá de varios factores como:

- Si los contenidos curriculares en cantidad y nivel de dificultad son adecuados, lo que facilitará el sentimiento de control del alumno para la asimilación del contenido. 
- Si los contenidos curriculares están ubicados dentro de un contenido global o más amplio facilitará la activación y localización de conocimientos previos para un aprendizaje significativo.

- Si los contenidos son novedosos, sorprendentes y variados generará en el alumno un interés y curiosidad por el nuevo contenido.

- Si son contenidos curriculares sensorialmente atractivos y acordes a las características del aprendiz despertarán interés exploratorio y curiosidad por aprender.

- Relacionar las actividades con la vida diaria para que vean el por qué y la utilidad de su estudio. La mayor parte de los alumnos no entienden para qué se estudian la mayoría de los contenidos de una materia. Esto crea el desánimo y la falta de interés por aprenderlos. Para que el alumno se sienta motivado hacia el aprendizaje de estos contenidos se le deben presentar de manera atractiva y relacionada con situaciones de la vida cotidiana.

Por ello, si se tienen en cuenta estos factores influyentes en la motivación del alumnado, a través del contenido se conseguirá que estos tengan mejores resultados y que las técnicas del profesorado puedan mejorarse. Además, el aprendizaje será constructivo para su futuro personal, social o educativo y no una simple tarea de memorizar.

\section{CONCLUSIONES}

Es vital que tanto los contenidos curriculares dados en clase como la docencia que realiza el profesor estén encaminados a crear alumnos competentes, en el plano académico, y en otros ámbitos de su vida. En este sentido, los alumnos prosociales puntúan significativamente más alto en metas de aprendizaje y logro. Así, es enorme la influencia recíproca del ámbito escolar y socio-familiar donde Wentzel (2005) establece, que las metas académicas y sociales de los estudiantes están relacionadas y la coordinación de estas contribuye de manera significativa al éxito escolar.

En lo referente a la importancia de los docentes en la práctica educativa, todos estos deben dedicar tiempo y esfuerzo suficiente para que los alumnos de este nivel educativo empiecen a convertirse en hábiles estudiantes; se les facilite la adquisición de las estrategias básicas del estudio, que contribuyan al desarrollo de un adecuada motivación y estrategias de aprendizaje, antes de que su rendimiento potencial se vea mermado por el desconocimiento o la falta de aplicación de dichas estrategias y motivaciones (Maquillón, 2011).

Por otro lado, las sucesivas reformas educativas realizadas durante las últimas décadas se han centrado en la motivación académica y en las calificaciones escolares de los estudiantes de ESO sin profundizar en el conocimiento relativo a la influencia que pueden tener diversos factores sociales o contextuales sobre las mismas. La existencia de interrelaciones entre el comportamiento prosocial y la motivación escolar, (Cándido, 2010) confirma lo argumentado por otros autores, quienes indican que la comunidad educativa debería atender a una variedad de factores interpersonales, incluyendo el establecimiento de relaciones sociales positivas o prosociales con los iguales, además de seguir enfatizando sobre diversas variables intrapersonales como la motivación escolar y el rendimiento académico (Pintrich, 2003). Dichas reformas educativas están sometidas a la realidad que establezca en Espacio Europeo de Educación Superior (EEES). 
En conclusión, se puede decir que pretende crear alumnos competentes socialmente, y no alumnos reproductores de lo que hicieron otros en el pasado.

\section{BIBLIOGRAFÍA}

Alonso Tapia, J. (2012). Differences between Immigrant and NationalStudents in Motivational Variables and Classroom-Motivational-Climate Perception. The spanish journal of psychology, 15 (1), 61-74.

Bernardo Carrasco, J. y Basterretche Baignol (2004). Técnicas y recursos para motivar a los alumnos. Madrid: Rialp, S.A..

Blumen S. (2008). Motivación sobredotación y talento: un desafío para el éxito. Revista de Psicología, 21 (1).

Carbonero M.A.; Martín-Antón L.J.; Román, J.M. y Repyo, N. (2010). Efecto de un programa de entrenamiento al profesorado en la motivación, clima de aula y estrategias de aprendizaje de su alumnado. Revista Iberomamericana de psicología y salud, 1 (2), 117-138.

García Bacete F.J. y Doménche Betoret, F. (2002). Motivación, aprendizaje y rendimiento escolar. Revista electrónica de motivación y emoción, 1 (6).

Inglés, C.J.; Martínez-González, A.E.; Valle, A.; García-Fernández, J.M. y RuizEsteban, C. (2010). Conducta prosocial y motivación académica en estudiantes españoles de Educación Secundaria Obligatoria. Universidad psychology, 10 (2), 451465.

Junco Herrera, I. (2009). La motivación en el proceso enseñanza-aprendizaje. Revista digital para profesionales de la enseñanza, 9, Julio 2009.

López Muñoz, L. (2004). La motivación en el aula. Pulso, 27, 95-107.

Maquillón Sánchez, J.J. y Hernández Pina, F. (2011). Influencia de la motivación en el rendimiento académico de los estudiantes de formación profesional. REIFOP, 14 (1), 81-100.

Mingorance Muley, A. (2010). ¿Cómo motivar al alumnado?, Revista digital innovación y experiencias educativas, 4.

Navarrete Ruiz de Clavijo, B. (2009). La motivación en el aula. Funciones del profesor para mejorar la motivación en el aprendizaje. Revista digital innovación y experiencias educativas, 15.

Román, J.M. (2008). Psicología de la instrucción. Valladolid. Universidad de Valladolid: Departamento de Psicología. 